\title{
LATE RESULTS OF REGIONAL BREAST CANCER SCREENING PROGRAM PERFORMED IN THE INTERIOR OF SÃO PAULO STATE, BRAZIL
}

Idam Oliveira-Junior¹, Edmundo C. Mauad'1, Bruno O. Fonseca', Anapaula H.U. Watanabe', René A.C.Vieira' 'Barretos Cancer Hospital - Barretos (SP), Brazil.

Objectives: Evaluation of the Barretos Regional Health Department (DRS V), São Paulo State results, after 15 years of its implantation. Methodology: The local Research Ethics Committee approved a retrospective study, of patients with breast cancer diagnosed at DRS-V/SP during a regional breast cancer-screening program. We evaluated the impact of mammographic screening in relation of clinical stages in three different periods: (1) Phase 1- 1999 to 2001, prior the beginning of the project; (2) Phase 2- 2003 to 2004, during the project implantation; (3) Phase 3- 2011 to 2016, in phase of consolidation. In Phase 3 , the rate of conservative mammary surgery, conservative axillary and maintenance of the mammary cosmese, was evaluated by biennium. Mammary cosmesis was considered the sum of the rate of conservative surgery and mastectomy with immediate reconstruction with prosthesis. The difference between the groups was analyzed by the chi-square test. Results: In the period prior to the introduction of the screening program, the rate of patients with early stage $(0+\mathrm{I})$ was $13 \%$. In, the first biennium (2003-2004) of implementation phase, at estimated population of 55,238 women, 17,964 women were screened ( $32.5 \%$ coverage) and 76 breast cancer patients were diagnosed. $45.4 \%$ of the women had never taken the exam in their lives. The rate of diagnosis of early tumors (EC $0+\mathrm{I})$ was $43.3 \%$. In Phase $3,55.6 \%$ of the patients were asymptomatic; and when the presence of breast symptoms was evaluated, it was observed that the early stage rate was $83.4 \%$ and $31.9 \%$, respectively, in the absence and presence of symptoms ( $\mathrm{p}<0.001)$. In the last biennium of Phase 3 (20152016 ) with an estimated 66,818 women, 593 cases were diagnosed (coverage of $47 \%$ ). In the different phases, the rate of early detection (EC $0+\mathrm{I}$ ) rose from $13.0 \%$ to $43.3 \%$ to $60.0 \%$, phase 1,2 and 3 , respectively ( $<<0.001$ ). The number of cases diagnosed per biennium in phase 3 was 165, 217 and 211, respectively. In this same phase the overall rate of conservative surgery was $68.1 \%$ in the breast, $74.5 \%$ in the armpit and $89.0 \%$ in the cosmese. Evaluating the first, second and third biennia of Phase 3, respectively: (1) Conservative surgery of $62.2 \%, 70.5 \%$ and $70.4 \%$; (2) Immediate reconstruction of $71.2 \%$, 54.8\% and 66.0\%; (3) Sentinel lymph node of 70.9, 76.8\% and 75.2\%; (4) Cosmese of 89.1\%, $88.1 \%$ and $89.9 \%$. Conclusion: The implementation of organized screening determines an increase in the number of early stage case and it is associated with an increase in the rate of conservative treatment and breast cosmesis. 